\title{
The comparison of acromiohumeral distance and scapular dyskinesis prevalence in females with and without rounded shoulder posture
}

\author{
Afsun Nodehi Moghadam*1 ${ }^{*}$, Elham Sarabadani Tafreshi ${ }^{1}$, Shima Abdollahi ${ }^{2}$, Enayatollah Bakhshi ${ }^{3}$
}

Received: 9 Jul 2019

Published: 11 Mar 2020

\section{Abstract}

Background: Altered scapular kinematics in individuals with rounded shoulder posture (RSP) may affect acromiohumeral distance (AHD). The purpose of this study was to compare AHD and scapular dyskinesis prevalence in individuals with and without RSP.

Methods: A total of 44 women (RSP, $n=21$; control, $n=23$ ) participated in the study. RSP was assessed by measuring acromion to table distance (ATD) in supine position and forward shoulder angle (FSA) using a camera and the AutoCAD software from the lateral side. According to these measurements, ATD more than $2.6 \mathrm{~cm}$ and FSA equal or more than $52^{\circ}$ were considered as RSP. AHD was measured via ultrasonography with the arm at rest and $90^{\circ}$ of active elevation in scapular plane (scaption). Also, scapular dyskinesis was assessed by Uhl yes/no rating system. Independent and paired t tests were used to compare AHD between and within groups' comparisons, respectively. Chi-square test was used to compare the scapular dyskinesis prevalence in individuals with or without RSP. The data were analyzed using the SPSS statistical software version 17. Significance level was set at 0.05

Results: There were no statistically significant differences in participants' AHD in rest position (dominant side, $\mathrm{p}=0.187$, nondominant side, $\mathrm{p}=0.144$ ) and $90^{\circ}$ scaption (dominant side, $\mathrm{p}=0.144$, nondominant side, $\mathrm{p}=0.182$ ) in each side between the 2 groups. Also, the results of chi-square test showed that there were not any significant differences in the prevalence of scapular dyskinesis during shoulder flexion (dominant side, $\mathrm{p}=0.653$, nondominant side, $\mathrm{p}=0.541$ ) and shoulder abduction (dominant side, $\mathrm{p}=0.141$, nondominant side, $\mathrm{p}=0.277$ ) in individuals with and without rounded shoulder postures.

Conclusion: According to our results, RSD cannot lead to reduced AHD and higher prevalence of scapular dyskinesis.

Keywords: Rounded shoulder posture, Acromiohumeral distance, Subacromial space, Scapular dyskinesis

Conflicts of Interest: None declared

Funding: None

*This work has been published under CC BY-NC-SA 1.0 license.

Copyright $\odot$ Iran University of Medical Sciences

Cite this article as: Nodehi Moghadam A, Sarabadani Tafreshi E, Abdollahi Sh, Bakhshi E. The comparison of acromiohumeral distance and scapular dyskinesis prevalence in females with and without rounded shoulder posture. Med J Islam Repub Iran. 2020 (11 Mar);34:19. https://doi.org/10.47176/mjiri.34.19

\section{Introduction}

Rounded shoulder posture (RSP) is characterized by altered scapular kinematics (scapular protraction, downward rotation, and anterior tilt) with increased cervical lordosis and upper thoracic kyphosis $(1,2)$. RSP is associated with alteration of scapular and glenohumeral orientation and kinematics due to muscle imbalance, such as shortening of pectorals and lengthening of rhomboids and middle and

Corresponding author: Dr Afsun Nodehi Moghadam, afsoonnodehi@gmail.com; af.nodehi@uswr.ac.ir

1. Department of Physiotherapy, University of Social Welfare and Rehabilitation Sciences, Tehran, Iran

2. Sajad Physiotherapy Clinic, Tehran, Iran

3. Department of Biostatistics and Epidemiology, University of Social Welfare and Rehabilitation Sciences, Tehran, Iran lower trapezius muscles (3). Accordingly, it was postulated that altered kinematics of the scapula or scapular dyskinesis (downward rotation, anterior tilt, and protraction) can place the anterior acromion in close proximity to rotator cuff tendons muscles and lead to impingement syndrome by decreasing the subacromial space (4-6).

Increased thoracic spine kyphosis (one of components

$\uparrow$ What is "already known" in this topic:

Rounded shoulder posture is associated with alteration in scapular and glenohumeral orientation and kinematics and muscle imbalance.

$\rightarrow$ What this article adds:

The result of this study showed that in individuals with rounded shoulder posture, alteration in scapular and glenohumeral orientation and kinematics may not lead to reduction of acromiohumeral distance. 
of RSP) has been shown to decrease scapular upward rotation, posterior tilting, and external rotation (7-9) . Also, radiographic images have shown that individuals with acquired or idiopathic thoracic hyperkyphosis, compared to nonkyphotic individuals, have a smaller acromiohumeral distance (AHD) (10). In addition to hyperkyphosis, the habitual slouched posture, which is common in everyday tasks, can be associated with anterior shift of the acromion process, protraction, downward rotation, and anterior tilt of scapula, which shortens the pectoralis minor muscle $(4,6,11)$. Short pectoralis minor muscle can restrict normal scapular movement during arm elevation, leading to narrowing the subacromial space $(4,11)$.

Kalra et al (12) showed that AHD did not differ between slouched and normal posture during $0^{\circ}$ and $45^{\circ}$ of active shoulder abduction. However, Kalra et al (12) induced the slouched posture artificially in a laboratory setting; therefore, it may not represent abnormal postures seen in individuals with RSP, which is characterized by protraction, internal rotation, anterior tilt, elevation, and abduction of scapula along with thoracic kyphotic posture.

Thigpen et al (6) showed that individuals with forward and rounded shoulder posture had significantly greater scapular internal rotation, upward rotation, and anterior tilt during loaded flexion and overhead reaching tasks. This mentioned altered scapular kinematics may contribute to impingement syndrome by decreasing the subacromial space. Generally, reduced AHD has been associated with impingement syndrome and rotator cuff disorders. Some studies with MRI and sonography reported a smaller AHD in patients with rotator cuff and impingement syndromes as compared to healthy shoulders (13-15). Thus, although the altered scapular kinematics has been shown in individuals with RSP, research has not shown a clear relationship between AHD and RSP. Therefore, alteration of scapular kinematics and spinal posture that have been shown in individuals with RSP may contribute to reduced AHD and impingement syndrome. We hypothesized that individuals with RSP would display a smaller AHD compared to individuals with normal shoulder girdle posture. Thus, the purpose of this study was to compare AHD and scapular dyskinesis prevalence in individuals with and without RSP.

\section{Methods}

\section{Participants}

Total of 44 asymptomatic participants were classified with either RSP $(n=21$, age $=21.95$ (2.63) years, and $\mathrm{BMI}=21.68(2.80))$ or without RSP $(\mathrm{n}=23$, age $=2.43$ (2.50) years, and $\mathrm{BMI}=21.72$ (2.42)) using forward shoulder angle measurement. The sample size was calculated with $80 \%$ confidence level and 5\% error probability for each group using similar studies for each group according to the sample size formula.

$$
n=\frac{\left(\left(z_{1-\frac{\alpha}{2}}+z_{1-\beta}\right)^{2} 2 \sigma_{p}^{2}\right)}{(d)^{2}}=\frac{\left((2 \cdot 8)^{2} 2 \times 0 \cdot 27\right)}{(0 \cdot 5)^{2}}
$$

Individuals were excluded if they had the following symptoms: (1) shoulder/neck pain; (2) presence of a history of instability, dislocation, or fracture of the shoulder joint; (3) previous surgery in the shoulder or neck region; (4) presence of neurologic symptoms; (5) cardiovascular disease; (6) genetic or congenital disorders; (7) pregnancy; and (8) movement limitation of the shoulder joint. The participants signed an informed consent form approved by the Ethical Committee of University of Social Welfare and Rehabilitation Sciences, Tehran, Iran (ethical code: USWR.REC.1393.216) and underwent a postural screening to identify RSP.

\section{Assessment of RSP}

RSP was assessed in 3 ways. At first, participants were assessed using a plumb line.

As viewed from the sagittal plane, rounded shoulder (anterior displacement of the acromion) was observed from left and right. When the acromial process was positioned anterior to the plumb line, the posture was considered as rounded shoulder. Then, the distance between the posterior border of the acromion and the table (acromion to table distance $=$ ATD) in the supine position was measured, and if this distance was more than $2.6 \mathrm{~cm}$, it was considered as rounded shoulder (16). Afterwards, the participants stood in a relaxed position, and looked straight. Posture was recorded from the lateral side using a camera; then, the forward shoulder angle (FSA) was measured using the AutoCAD software (Fig. 1). According to this scale, shoulder angle equal or more that $52^{\circ}$ is considered as rounded shoulder (6).

\section{Measurement of AHD using Ultrasonography}

In this study, the AHD of dominant and nondominant arm was measured via ultrasonography (Mindray device model dp6600 with a linear probe and $12 \mathrm{MHz}$ frequency) in 2 arm positions: the arm at rest and $90^{\circ}$ active scaption ( $40^{\circ}$ front of the frontal plane). The participants sat in an upright position on a wooden chair, while their feet were in contact with the floor in relaxed position. They were asked to maintain an upright posture with double limb support. The linear probe was placed in line with the supraspinatus fibers in the middle point of the acromiohumeral joint, and the AHD distance was measured from below the acromion bone perpendicular to the humeral head in a resting position, and then in $90^{\circ}$ elevation of the scapula $(13,17)$ (Fig. 2: A, B, C, D).

\section{Scapular dyskinesis test}

The Uhl yes/no rating system was used to evaluate scapular dyskinesis (18). Participants were asked to elevate the arms and to lower them over a 3 -second count in sagittal and frontal planes in a relaxed standing position with the thumbs pointed up. Presence or absence of scapular dyskinesis (winging and/or Dysrhythmia) is observed during 5 repetitions of flexion and abduction with grasping dumbbells according to body weight $(1 \mathrm{~kg}$ for those weighing less than $60 \mathrm{~kg}$ and $2 \mathrm{~kg}$ for those weighing 60 $\mathrm{kg}$ or more) $(18,19)$.

Prior to data collection, test-retest intersession reliability of ATD, AHD, and FSA were obtained for 10 participants 


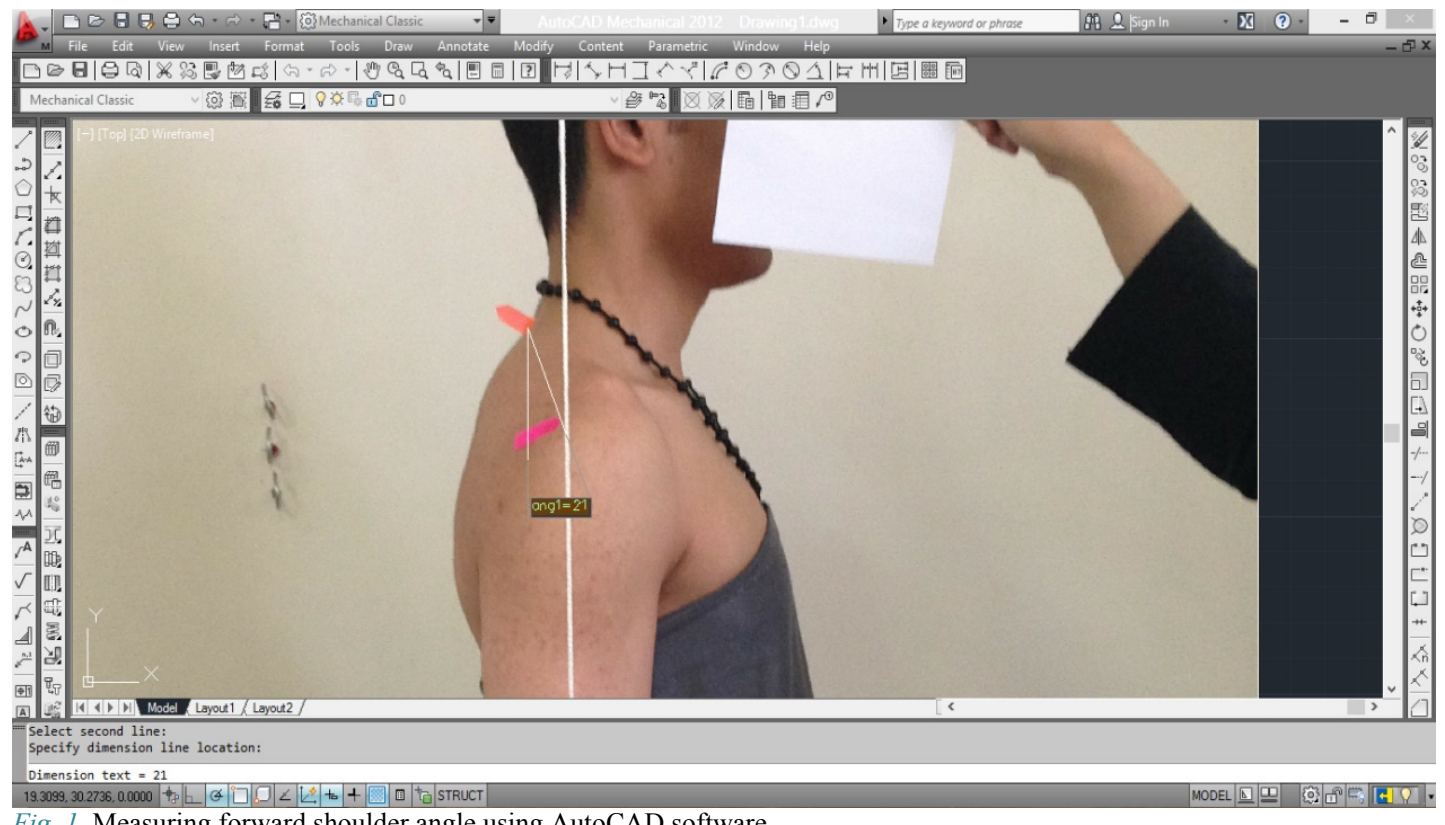

Fig. 1. Measuring forward shoulder angle using AutoCAD software

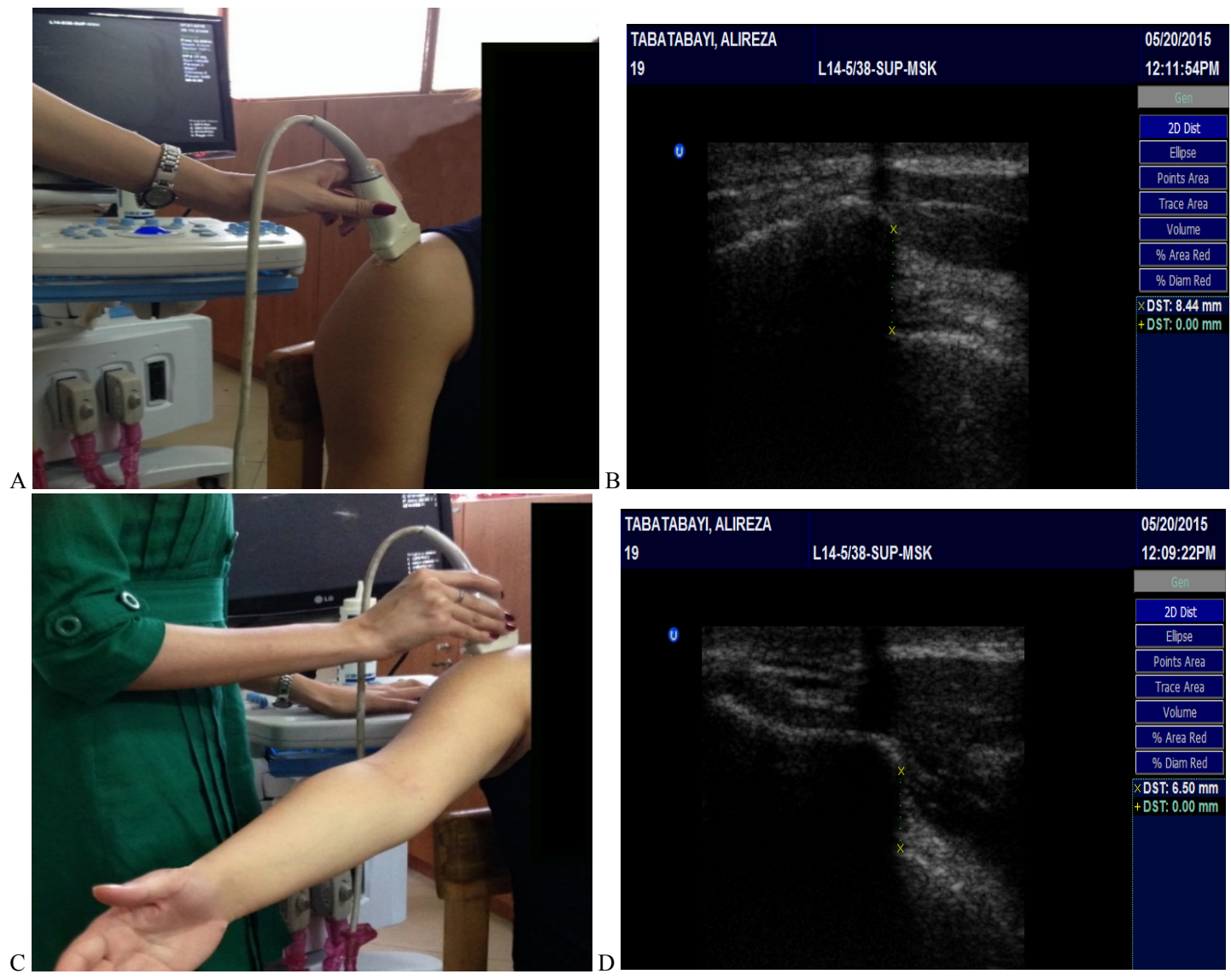

Fig. 2. Measurement of AHD: A, B: Arm resting next to the body, C, D Arm in $90^{\circ}$ abduction in the plane of scapula

according to the methodology described above. To test the reliability of the scapular dyskinesis test, assessment was videotaped and several days later, the tape was watched and scored again by those blinded to the previous measurement results. ICC results were reported with $95 \%$ confident interval and reliability was regarded as very low to very high. Also, 10 participants entered the methodological study, assessments were repeated at the same day with 1-hour interval in the right side for all participants by the same operator and data were analyzed by a doublemeasurement. 


\section{Statistical analysis}

Data were analyzed using SPSS statistical software version 17. Significance level was considered at 0.05. Demographic characteristics were described in mean and standard deviation. Intra Class Correlation (ICC), Standard Error of Measurement (SEM), and Minimum Detectable Change (MDC) were used to assess the relative and absolute reliability of the study measurements. KolmogorovSmirnov test was used to assess the distribution of the variables. To assess the equality of variances for a variable calculated for the 2 groups, Levene's test was used. To investigate group differences, independent $t$ test was used for the quantitative variables. Also, chi-square test was used to determine whether there was a significant difference between the scapular dyskinesis prevalence in individuals with or without RSP. Significance level was set at 0.05 .

\section{Results}

The ICCs, SEMs, and MDC are presented in Table 1. According to Table 1, the ICCs for ADH, FSA, ATD, and scapular dyskinesis are considered as high reproducibility.

The means (SD) of age and body mass index (BMI) of the individuals with or without RSP were 22.95 (2.63) years, $21.68(2.80) \mathrm{kg} / \mathrm{m}^{2}$, and 22.43 (2.50) years, 21.72 (2.42) $\mathrm{kg} / \mathrm{m}^{2}$, respectively. There were no statistically significant differences in participants' age $(p=0.507)$ and body mass index $(p=0.957)$ between the 2 groups. Table 2 demonstrates the comparison of demographic characteristics, postural variables, and AHD in dominant and nondominant sides of individuals with and without rounded shoulder postures. Moreover, there were no statistically significant differences in participants' AHD in rest position (dominant side, $\mathrm{p}=0.187$; nondominant side, $\mathrm{p}=0.144$ ) and $90^{\circ}$ abduction (dominant side, $\mathrm{p}=0.137$, nondominant side, $\mathrm{p}=0.182$ ) in each side between two groups (Table 2).

The results of paired samples $t$ test showed no significant differences in AHD in rest position (control group, $\mathrm{p}=0.272$; RSP, $\mathrm{p}=0.164$ ) and $90^{\circ}$ abduction (control group, $\mathrm{p}=0.959$; RSP, 0.305 ) between the 2 dominant and nondominant sides. Also, the results of chi-square test showed no significant differences in the prevalence of scapular dyskinesis during shoulder flexion (dominant side, 0.653; nondominant side, $\mathrm{p}=0.541$ ) and shoulder abduction (dominant side, $\mathrm{p}=0.141$; nondominant side, $\mathrm{p}=0.277$ ) in individuals with and without rounded shoulder postures (Table 3).

Table 1. The intraclass correlation coefficients (ICC), standard errors of measurement (SEM), and minimum detectable change values for assessment of intratester reliability of measured variables

\begin{tabular}{|c|c|c|c|}
\hline Variable & ICC & SEM & MDC \\
\hline ATD & 0.97 & 0.42 & 1.16 \\
\hline FSA & 0.97 & 4.3 & 11.86 \\
\hline AHD in rest position & 0.93 & 0.43 & 1.18 \\
\hline AHD in $90^{\circ}$ abduction & 0.94 & 0.49 & 1.35 \\
\hline Scapular dyskinesis test in flexion & 0.83 & 0.33 & 0.91 \\
\hline Scapular dyskinesisin abduction & 0.84 & 0.40 & 1.1 \\
\hline
\end{tabular}

$\mathrm{AHD}=$ Acromiohumeral distance; FSA $=$ Forward shoulder angle, $\mathrm{ATD}=$ Distance between acromion and the table, $\mathrm{MDC}=\mathrm{Minimum}$ detectable change.

Table 2. The comparison of demographic characteristics, postural variables, and AHD in dominant and nondominant sides of individuals with and without rounded shoulder postures

\begin{tabular}{|c|c|c|c|c|c|c|}
\hline \multirow[t]{2}{*}{ Variable } & \multicolumn{2}{|c|}{ Mean (SD) } & \multirow[t]{2}{*}{ P-value } & \multirow{2}{*}{$\begin{array}{c}\text { Observed } \\
\text { Power }^{\mathrm{m}}\end{array}$} & \multicolumn{2}{|c|}{$95 \%$ of confidence interval of difference } \\
\hline & Control $(n=23)$ & $\operatorname{RSP}(n=21)$ & & & Lower & Upper \\
\hline Age (years) & $22.43(2.50)$ & $22.95(2.62)$ & 0.507 & 0.100 & -2.07 & 1.04 \\
\hline $\operatorname{BMI}\left(\mathrm{kg} / \mathrm{m}^{2}\right)$ & $21.72(2.42)$ & $21.68(2.80)$ & 0.957 & 0.050 & -1.55 & 1.64 \\
\hline D-FSA (degree) & $18.83(3.07)$ & $55.19(4.25)$ & $<0.001$ & 1.000 & -38.64 & -34.07 \\
\hline ND-FSA (degree) & $17.30(3.79)$ & $51.05(4.31)$ & $<0.001$ & 1.000 & -36.23 & -31.25 \\
\hline D-ATD (centimeters) & $2.33(0.24)$ & $5.43(1.03)$ & $<0.001$ & 1.000 & -3.57 & -2.61 \\
\hline ND-ATD (centimeters) & $2.22(0.24)$ & $5.07(1.03)$ & $<0.001$ & 1.000 & -3.32 & -2.37 \\
\hline D-AHD (rest) (Millimeters) & $9.64(1.54)$ & $10.56(2.73)$ & 0.187 & 0.272 & -2.29 & 0.46 \\
\hline D-AHD ( $90^{\circ}$ abd) (Millimeters) & $7.34(1.26)$ & $8.16(2.16)$ & 0.137 & 0.332 & -1.92 & 0.27 \\
\hline ND-AHD (rest) (Millimeters) & $9.88(1.81)$ & $10.88(2.52)$ & 0.144 & 0.317 & -2.35 & 0.35 \\
\hline ND-AHD ( $\left.90^{\circ} \mathrm{abd}\right)$ (Millimeters) & $7.33(1.09)$ & $7.97(1.88)$ & 0.182 & 0.276 & -1.59 & 0.31 \\
\hline
\end{tabular}

$\mathrm{AHD}=$ Acromiohumeral distance; FSA = Forward shoulder angle, ATD = Distance between the acromion and the table, $\mathrm{D}=\mathrm{Dominant}$ side, ND $=$ nondominant side, $\mathrm{BMI}=$ Body Mass Index. Mean difference

Table 3. The prevalence of scapular dyskinesis during shoulder flexion and abduction in dominant and nondominant sides of individuals with and without rounded shoulder postures.

\begin{tabular}{|c|c|c|c|c|c|}
\hline \multicolumn{6}{|c|}{ Scapular dyskinesis: N (\%within group) } \\
\hline \multicolumn{2}{|c|}{ Shoulder elevation planes } & \multicolumn{2}{|c|}{ Dominant side } & \multicolumn{2}{|c|}{ Non dominant side } \\
\hline & & Control & RSP & Control & RSP \\
\hline \multirow[t]{2}{*}{ Flexion } & Yes & $3(13 \%)$ & $4(19 \%)$ & $5(21.7 \%)$ & $5(23.8 \%)$ \\
\hline & No & $20(87 \%)$ & $17(81 \%)$ & $18(78.3 \%)$ & $16(76.2 \%)$ \\
\hline \multirow[t]{2}{*}{ Abduction } & Yes & $1(4.3 \%)$ & $22(95.7 \%)$ & $2(8.7 \%)$ & $4(19 \%)$ \\
\hline & No & $2(9.5 \%)$ & $19(90.5 \%)$ & $21(91.3 \%)$ & $17(81 \%)$ \\
\hline
\end{tabular}




\section{Discussion}

According to our results, no significant differences were found for the AHD (arm in rest and $90^{\circ}$ elevation in scapular plane) and the scapular dyskinesis prevalence between individuals with and without rounded shoulder postures.

RSP is characterized by protraction, internal rotation, anterior tilt, elevation, and abduction of scapula along with thoracic kyphotic posture $(1,2)$. It is assumed that altered kinematics of the scapula or scapular dyskinesis (downward rotation, anterior tilt, and protraction) may contribute to impingement syndrome by decreasing the subacromial space (4-6). There are 2 common mechanisms for explaining the causes of decreasing the subacromial space: (1) superior humeral head translation and (2) altered scapular kinematics (scapular downward rotation, anterior tilt, and protraction) (20). Superior humeral translation subsequent to rotator cuff weakness or fatigue has been shown in previous studies (20). However, no consensus exists based on the literature about the altered scapular kinematics contributions on reducing subacromial space. In agreement to our results, Chopp et al (21) in a simulation analysis study found that humeral head translation plays a larger role in reducing subacromial space width, while altered scapular kinematics would increase this space. Therefore, they suggested that the altered scapular kinematics (scapular dyskinesis) had minimal effects on subacromial space width, so it can be considered as an impingement protective strategy (21).

Wang et al (17) investigated the thickness of the biceps and supraspinatus tendons as well as the width of the subacromial space by sonography (in frontal and scapular planes) in the 2 groups of baseball players with and without shoulder injury and a group of healthy nonathletes. Results showed that the biceps and supraspinatus tendons' thicknesses and the AHD (in resting and $90^{\circ}$ abduction positions) were higher in the athletes compared to the nonathletic group (17). However, in a study, using MRI, Graichen et al (22) found that the subacromial space was reduced between the $30^{\circ}-90^{\circ}$ abduction. The increased AHD found in the Wang et al (17) study may be a preventive mechanism to prevent further injuries.

Also, Gumina et al (10) showed that individuals with thoracic hyperkyphosis greater than 50 had a smaller AHD (with the arm at rest) as measured on radiographic images, compared to nonkyphotic individuals. RSP is not analogous to hyper kyphosis. Increased thoracic curvature is along with altered scapular kinematics in individuals with RSP, while Gumina et al evaluated individuals with hyperkyphosis and measured AHD in resting position only.

In the present study, the ultrasonographic assessment of AHD was down in $0^{\circ}$ and $90^{\circ}$ shoulder arm elevation. During arm elevation, the humeral head displaces superiorly until $90^{\circ}$ arm elevation, which leads to reducing subacromial space width. On the other hand, patients with impingement syndrome may have a painful arc of motion near $90^{\circ}$ of humeral elevation, because at this range the forces on rotator cuff muscle are the highest. Kalra et al showed that AHD did not differ between artificially in- duced slouched posture and normal posture during $0^{\circ}$ and $45^{\circ}$ of active shoulder abduction.

In agreement with our results, Mackenzie et al (20) in their study to determine if a relationship exists between extrinsic factors and AHD found that there were not any correlation between scapular rotation and thoracic curvature with AHD. Also, according to Thomas et al (23) study, no correlations were found between AHD and scapular upward rotation at either the $0^{\circ}$ or $90^{\circ}$ shoulder elevation, suggesting no change in AHD with increased scapular upward rotation.

Reduced subacromial space was proposed as a predictive factor in subacromial impingement syndrome. However, the results of some studies that assessed the subacromial space width in pathologic populations had conflicting results. The results of Hebert et al (15) demonstrated a reduced AHD in the impingement group compared with the control group at the $80^{\circ}, 90^{\circ}$, and $110^{\circ} \mathrm{ab}-$ duction. However, Desmeules et al (24) did not find any significant difference between the impingement group and the healthy control group in AHD. Therefore, a lack of difference in AHD between the 2 groups in our study may be due to the fact that the principal factor in the change in subacromial space was the displacement of the humeral head, and not the direction of the scapula. It is also possible that if a change in scapular position or motions occurs, it is more like a protective or compensatory mechanism to offset superior humeral head migration to decrease the pressure on the soft tissues passing through the subacromial space (21). Also, according to Gumina et al (25) study that evaluated AHD in elderly twins, it was found that AHD is primarily genetically determined and only its margins may be influenced by external factors. Therefore, it seems that the determinants of subacromial space width appear to be multifactorial and possibly populationspecific and there are additional factors involved in determining AHD, which should be considered. Additional studies are needed to confirm the determinants of subacromial space width.

There were some limitations in this study that should be taken into account. First, AHD was measured only in the $0^{\circ}$ and the $90^{\circ}$ shoulder elevation positions; therefore, the results could not be generalized to other angles. Second, our sample was a group of asymptomatic females, so applying these results to the symptomatic and male population should be done with caution. Lastly, the assessment of scapular movement and position were based on clinical judgment rather than 3-dimensional motion analysis systems.

\section{Conclusion}

There were no statistically significant differences in participants' AHD in rest position and $90^{\circ}$ abduction in each side between individuals with or without RSP. Also, there were not any significant differences in scapular dyskinesis prevalence during shoulder flexion and scaption in dominant and nondominant sides of individuals with and without rounded shoulder posture. 


\section{Conflict of Interests}

The authors declare that they have no competing interests.

\section{References}

1. Wong CK, Coleman D, diPersia V, Song J, Wright D. The effects of manual treatment on rounded-shoulder posture, and associated muscle strength. J Bodyw Mov Ther. 2010;14(4):326-33.

2. Lee JH, Cynn HS, Yoon TL, Ko CH, Choi WJ, Choi SA, et al. The effect of scapular posterior tilt exercise, pectoralis minor stretching, and shoulder brace on scapular alignment and muscles activity in subjects with round-shoulder posture. J Electromyogr Kinesiol. 2015;25(1):107-14.

3. Singla D, Veqar Z. Association Between Forward Head, Rounded Shoulders, and Increased Thoracic Kyphosis: A Review of the Literature. J Chiropr Med. 2017;16(3):220-9.

4. Ludewig PM, Cook TM. Alterations in shoulder kinematics and associated muscle activity in people with symptoms of shoulder impingement. Phys Ther. 2000;80(3):276-91.

5. McClure PW, Bialker J, Neff N, Williams G, Karduna A. Shoulder function and 3-dimensional kinematics in people with shoulder impingement syndrome before and after a 6-week exercise program. Phys Ther. 2004;84(9):832-48

6. Thigpen CA, Padua DA, Michener LA, Guskiewicz K, Giuliani C, Keener JD, et al. Head and shoulder posture affect scapular mechanics and muscle activity in overhead tasks. J Electromyogr Kinesiol. 2010;20(4):701-9.

7. Finley MA, Lee RY. Effect of sitting posture on 3-dimensional scapular kinematics measured by skin-mounted electromagnetic tracking sensors. Arch Phys Med Rehabil. 2003;84(4):563-8.

8. Kebaetse M, McClure P, Pratt NA. Thoracic position effect on shoulder range of motion, strength, and three-dimensional scapular kinematics. Arch Phys Med Rehabil. 1999;80(8):945-50.

9. Lewis JS, Green A, Wright C. Subacromial impingement syndrome: the role of posture and muscle imbalance. J Shoulder Elbow Surg. 2005;14(4):385-92.

10. Gumina S, Di Giorgio G, Postacchini F, Postacchini R. Subacromial space in adult patients with thoracic hyperkyphosis and in healthy volunteers. Chir Organi Mov. 2008;91(2):93-6.

11. Lee JH. Effects of forward head posture on static and dynamic balance control. J Phys Ther Sci. 2016;28(1):274-7.

12. Kalra N, Seitz AL, Boardman ND, 3rd, Michener LA. Effect of posture on acromiohumeral distance with arm elevation in subjects with and without rotator cuff disease using ultrasonography. J Orthop Sports Phys Ther. 2010;40(10):633-40.

13. Azzoni R, Cabitza P, Parrini M. Sonographic evaluation of subacromial space. Ultrasonics. 2004;42(1-9):683-7.

14. Graichen $\mathrm{H}$, Bonel $\mathrm{H}$, Stammberger $\mathrm{T}$, Haubner M, Rohrer $\mathrm{H}$, Englmeier KH, et al. Three-dimensional analysis of the width of the subacromial space in healthy subjects and patients with impingement syndrome. AJR Am J Roentgenol. 1999;172(4):1081-6.

15. Hebert LJ, Moffet H, Dufour M, Moisan C. Acromiohumeral distance in a seated position in persons with impingement syndrome. J Magn Reson Imaging. 2003;18(1):72-9.

16. Sahrmann S. Diagnosis and treatment of movement impairment syndromes: Elsevier Health Sciences; 2001.

17. Wang HK, Lin JJ, Pan SL, Wang TG. Sonographic evaluations in elite college baseball athletes. Scand J Med Sci Sports. 2005;15(1):2935 .

18. Uhl TL, Kibler WB, Gecewich B, Tripp BL. Evaluation of clinical assessment methods for scapular dyskinesis. Arthroscopy. 2009;25(11):1240-8.

19. McClure P, Tate AR, Kareha S, Irwin D, Zlupko E. A clinical method for identifying scapular dyskinesis, part 1: reliability. J Athl Train. 2009;44(2):160-4.

20. Mackenzie TA, Herrington L, Funk L, Horsley I, Cools A. Relationship between extrinsic factors and the acromio-humeral distance. Man Ther. 2016;23:1-8.

21. Chopp JN, Dickerson CR. Resolving the contributions of fatigueinduced migration and scapular reorientation on the subacromial space: an orthopaedic geometric simulation analysis. Hum Mov Sci. 2012;31(2):448-60.

22. Graichen H, Hinterwimmer S, von Eisenhart-Rothe R, Vogl T, Englmeier KH, Eckstein F. Effect of abducting and adducting muscle activity on glenohumeral translation, scapular kinematics and subacromial space width in vivo. J Biomech. 2005;38(4):755-60.

23. Thomas SJ, Swanik CB, Kaminski TW, Higginson JS, Swanik KA, Nazarian LN. Assessment of subacromial space and its relationship with scapular upward rotation in college baseball players. J Sport Rehabil. 2013;22(3):216-23.

24. Desmeules F, Minville L, Riederer B, Cote CH, Fremont P. Acromio-humeral distance variation measured by ultrasonography and its association with the outcome of rehabilitation for shoulder impingement syndrome. Clin J Sport Med. 2004;14(4):197-205.

25. Gumina S, Arceri V, Fagnani C, Venditto T, Catalano C, Candela V, et al. Subacromial Space Width: Does Overuse or Genetics Play a Greater Role in Determining It? An MRI Study on Elderly Twins. J Bone Joint Surg Am. 2015;97(20):1647-52. 